Max-Planck-Institut für demografische Forschung

Max Planck Institute for Demographic Research

Konrad-Zuse-Strasse 1 - D-18057 Rostock - GERMANY

Tel +49 (0) 3812081 - 0; Fax +49 (0) 3812081 - 202;

http://www.demogr.mpg.de

MPIDR WORKING PAPER WP 2012-001

JANUARY 2012

\title{
Diversität von Familienformen \\ in Ost- und Westdeutschland
}

\author{
Sonja Bastin (bastin@demogr.mpg.de) \\ Michaela Kreyenfeld (kreyenfeld@demogr.mpg.de) \\ Christine Schnor (schnor@demogr.mpg.de)
}

(C) Copyright is held by the authors.

Working papers of the Max Planck Institute for Demographic Research receive only limited review. Views or opinions expressed in working papers are attributable to the authors and do not necessarily reflect those of the Institute. 


\section{Diversität von Familienformen in Ost- und Westdeutschland}

Sonja Bastin, Michaela Kreyenfeld und Christine Schnor

\section{Zusammenfassung}

In diesem Beitrag wird die Diversität der Lebensformen in Deutschland mit den Daten der ersten Welle des Beziehungs- und Familienpanels (pairfam) aus den Jahren 2008/09 dargestellt. Die Analysen wurden auf die Kohorten 1971-1973 beschränkt. Diese Jahrgänge haben im wiedervereinten Deutschland das Erwachsenenalter erreicht und wesentliche familiale Übergänge, wie die Geburt des ersten Kindes, mittlerweile vollzogen. Der Schwerpunkt der Analysen liegt auf der Dynamik, die Lebensformen nach der Familiengründung erfahren. Es zeigen sich große Ost-West-Unterschiede in den Familienbildungsmustern. Ostdeutsche Befragte sind häufiger unverheiratet bei der Geburt ihres ersten Kindes und heiraten zudem seltener nach der Familiengründung als westdeutsche Befragte. Ostdeutsche Frauen trennen sich insbesondere im ersten Lebensjahr ihres Kindes häufiger als westdeutsche Mütter. Zudem ist mit mehr als zehn Prozent der Anteil an Personen, die bereits bei der Geburt des ersten Kindes keinen Partner (mehr) haben, in Ostdeutschland auffällig hoch. Betrachtet man Trennungsrisiken nach der Lebensform zum Zeitpunkt der Geburt des ersten Kindes zeigt sich, dass nichteheliche Lebensgemeinschaften ein höheres Trennungsrisiko aufweisen als eheliche Lebensgemeinschaften. Im Ost-West-Vergleich sind ostdeutsche Ehen instabiler. Nichteheliche Lebensgemeinschaften weisen in Ostdeutschland hingegen eine niedrigere Trennungswahrscheinlichkeit auf als in Westdeutschland.

Schlüsselbegriffe: Familie, Lebensform, Trennung, Scheidung, Ost- und Westdeutschland 


\title{
Diversity of Family Forms in Eastern and Western Germany
}

Sonja Bastin, Michaela Kreyenfeld und Christine Schnor

\begin{abstract}
In this paper data from the first wave of the German family panel (pairfam) is used to study the diversity of living arrangements in Germany. The analyses are restricted to the birth cohorts 1971-1973. These cohorts have entered adulthood after unification, but made significant transitions, like the birth of their first children, until interview in 2008/09. The investigation focuses on the union dynamics after the birth of the first child. We find significant differences in family formation patterns between eastern and western Germany. Respondents in eastern Germany are more often unmarried than western German respondents when the first child is born. Also after family formation, western Germans remain more likely to marry than eastern Germans. Separation rates are higher in eastern Germany in the immediate year after childbirth. A striking feature of the eastern German pattern is that a large fraction of roughly ten percent of the respondents does not have a partner (anymore) when the first child is born. We have also studied how partnership status at first birth relates to separation rates. In line with other studies, we find that cohabiting unions are less stable than marital unions. Comparing behavior in eastern and western Germany, we find that marital unions in the West are more stable than in the East. However, cohabiting unions in the eastern part of Germany display lower separation rates than non-marital unions in the western states.
\end{abstract}

Keywords: Family, Living Arrangement, Separation, Germany, Eastern Germany, Western Germany 


\section{Einleitung}

Unsere Vorstellung vom familialen Wandel ist vor allem von der Idee geprägt, dass die Familie in Deutschland - wie auch jene in anderen westeuropäischen Ländern - seit den 1960er Jahren durchgreifenden Veränderungen unterworfen ist. Der radikale Rückgang der Heirats- und Fertilitätsraten, der Anstieg der Scheidungsintensität und die Zunahme von nichtehelichen Lebensgemeinschaften und anderen Lebensformen jenseits der bürgerlichen Kleinfamilie werden dabei routinemäßig unter die Begrifflichkeiten der Pluralisierung, Destandardisierung oder Deinstitutionalisierung gefasst oder auch mit wertenden Begriffen wie dem „Zerfall der Familie“ belegt (Tyrell 1988; Nave-Herz 1997; Strohmeier 1993; Wagner und Franzmann 2000; Peuckert 2008). In der internationalen Forschung stehen häufig modernisierungstheoretische Überlegungen im Vordergrund, um den familialen Wandel zu beschreiben, wobei insbesondere unter den Begrifflichkeiten des „zweiten demographischen Übergangs“ die Vorstellung eines gleichgerichteten Prozesses der zunehmenden Modernisierung und Diversifizierung der Familienstrukturen vertreten wird (Van de Kaa 1987; Lesthaeghe 1992).

Eine Vielzahl von empirischen Studien sind für Deutschland (Strohmeier 1993; Schneider et al. 1998; Wagner und Franzmann 2000) wie auch für andere europäische Länder (Elzinga und Liefbroer 2007; Fokkema und Liefbroer 2008) vorgelegt worden, die diesen Wandel darstellen. Zudem publiziert die amtliche Statistik regelmäßig Kennziffern, in denen auf Basis der Daten des Mikrozensus die Lebens- und Familienformen abgebildet werden (Statistisches Bundesamt et al. 2011). Trotz dieser Fülle an empirischen Evidenzen sind bislang nur selten Versuche unternommen worden, die Dynamik der Lebens- und Familienformen im Längsschnitt abzubilden (Brüderl 2004). Ziel dieses Beitrags ist es, 
diese Diversität der Lebensformen im Längsschnitt zu erfassen, indem die Dynamik, welcher Lebensformen nach der Familiengründung unterworfen sind, dargestellt wird.

Als Datenbasis dienen die erste Welle des deutschen Beziehungs- und Familienpanels (pairfam) (Huinink et al. 2011) sowie der ostdeutschen Zusatzstichprobe DemoDiff (Kreyenfeld et al 2011). ${ }^{1}$ Die für Deutschland repräsentative Panelstudie befragt seit 2008/2009 jährlich Personen der Geburtskohorten 1971-1973, 1981-1983 und 1991-1993. Durch die retrospektive Erhebung von Partner- und Elternschaftsbiografien können die gesamten Lebensläufe dieser Kohorten abgebildet werden. Da jedoch die Kohorten 19811983 und 1991-93 noch relativ jung sind und damit viele Übergänge im familialen Lebenslauf noch nicht erfahren haben, konzentrieren wir uns in diesem Beitrag auf die Lebensläufe der Geburtsjahrgänge 1971-73. Diese Kohorten sind zum Zeitpunkt der Befragung zwischen 35 und 39 Jahre alt. Eine Besonderheit dieser Jahrgänge ist, dass sie das Erwachsenenalter im wiedervereinigten Deutschland durchlebt, jedoch die ostdeutschen Befragten dieser Kohorten ihre Sozialisation noch in der DDR erfahren haben (Mayer und Schulze 2009). Aus diesem Grund und auf Basis der Tatsache, dass sich familiale Verhaltensweisen in Ost- und Westdeutschland weiterhin unterscheiden, führen wir alle Analysen getrennt für Ost- und Westdeutschland durch.

Der Beitrag ist wie folgt aufgebaut. In Teil 2 werden auf Basis einfacher Kreuztabellen die Lebens- und Familienformen in Ost- und Westdeutschland nach Alter dargestellt. Zur Klassifikation von Lebens- und Familienformen werden, neben dem Vorliegen einer Elternschaft, die Dimensionen Familienstand, Haushaltskontext und Paarbeziehung

1 Wenn im Folgenden das „Beziehungs- und Familienpanel” genannt wird, sind damit die Daten des Projekts „Panel Analysis of Intimate Relationships and Family Dynamics“ (pairfam) sowie des Projekts "Demographic Differences in Life Course Dynamics in Eastern and Western Germany" (DemoDiff) gemeint. 
herangezogen. Teil 3 fokussiert die Dynamik der Lebensformen nach der Geburt des ersten Kindes. Ein erheblicher Anteil der Befragten, insbesondere in Ostdeutschland, lebt in einer nichtehelichen Lebensgemeinschaft, wenn das erste Kind geboren wird. Auf Basis von Zeitdaueranalysen wird dargestellt, ob diese Lebensform von Dauer ist oder die Personen auch noch nach der Familiengründung heiraten. Zudem wird das Trennungsverhalten nach der Familiengründung untersucht. Teil 5 liefert eine abschließende Betrachtung.

\section{Lebensformvariationen der Geburtskohorten 1971-1973}

\subsection{Versuch einer Taxonomie der Lebensformen}

Der Versuch einer Kategorienbildung von Lebens- und Familienformen muss sich zwangsläufig an einem Lebensformen- und Familienbegriff orientieren. Während mittlerweile einheitlich das Kind als konstituierendes Moment der Familie definiert wird, gibt es bislang kein einheitliches Vorgehen zur Klassifikation von Lebensformen. In der amtlichen Statistik dominiert weiterhin eine auf den Haushalt beschränkte Definition der Lebensformen. Demgegenüber wird in der familiensoziologischen Forschung durch die Verfügbarkeit neuerer Datensätze vermehrt der Versuch unternommen, auch die haushaltsübergreifenden Strukturen zu erfassen, indem „Living-Apart-Together Beziehungen“ betrachtet oder die komplexen Wohnverhältnisse abgebildet werden, in denen Stieffamilien leben (Feldhaus und Schlegel 2011; Feldhaus und Huinink 2011; Schneider et al. 2001). Wir folgen diesem Vorgehen und berücksichtigen haushaltsübergreifende Strukturen in der Weise, dass wir die drei Dimensionen Familienstand, Haushaltskontext und Vorliegen einer Paarbeziehung heranziehen, um 
Lebensformen zu kategorisieren (siehe auch Huinink und Konietzka 2007: 39). Der Familienstand gibt dabei den Grad der rechtlichen Institutionalisierung einer Partnerschaft wieder und unterscheidet die Dimensionen ledig, verheiratet und geschieden bzw. verwitwet. $^{2}$ Der Haushaltskontext zeigt an, ob die Person mit einem Partner zusammen in einem Haushalt lebt (ohne Koresidenz, mit Koresidenz). Die Dimension der Paarbeziehung beinhaltet, ob eine Partnerschaft zu einer Person vorliegt (kein Partner, Partner).

Aus den drei Dimensionen Familienstand, Haushaltskontext und Paarbeziehung ergeben sich 9 logische Kategorien, wobei einige der Kategorien dünn besetzt sind. Abbildung 1 verdeutlicht diesen Umstand auf Basis der ersten Welle des Beziehungs- und Familienpanels, welche auf die Stichprobe der Frauen und Männer der Geburtskohorten 1971-73 reduziert wurde. ${ }^{3}$ Die Verwitweten werden in der Darstellung zusammen mit den Geschiedenen ausgewiesen, da die Befragten der Kohorten 1971-73 noch relativ jung sind und es bislang kaum verwitwete Personen in diesen Geburtsjahrgängen gibt $(\mathrm{N}=15)$.

2 Personen in eingetragenen Partnerschaften wurden als verheiratet betrachtet.

3 Die gesamte Stichprobe des Beziehungs- und Familienpanels umfasst 4.792 Befragte der Kohorten 1971-1973. 145 Personen wurden aus den Analysen ausgeschlossen, da für diese Personen keine validen Fertilitäts- oder Partnerschaftsbiographien oder keine Angaben zum Wohnort zum Befragungszeitpunkt vorlagen. 
Abbildung 1: Lebensformen der Geburtskohorten 1971-73 zum Zeitpunkt der Befragung nach Familienstand, Paarbeziehung und Haushaltskontext in absoluten Zahlen

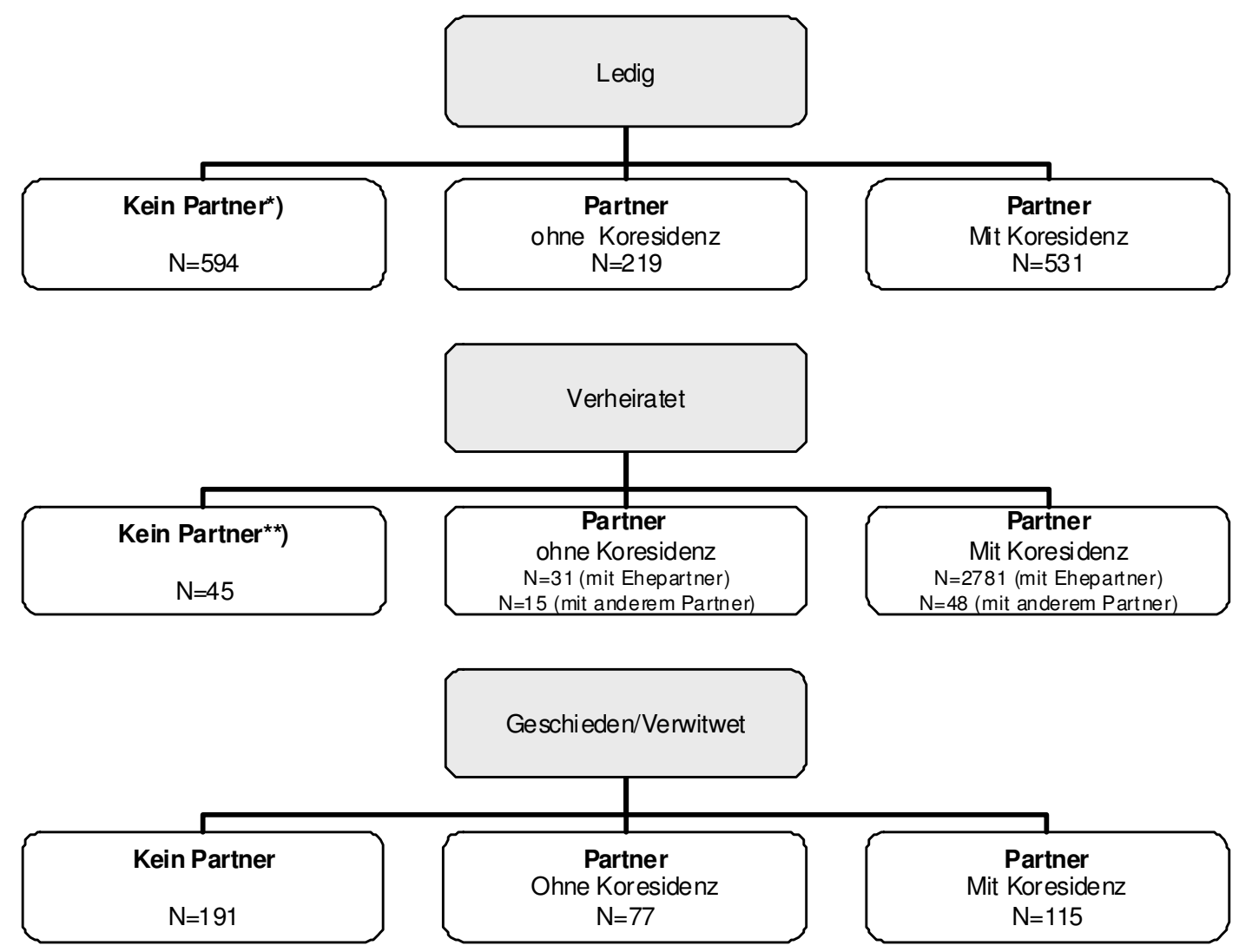

Anmerkung: *) 6 Fälle führen einen gemeinsamen Haushalt mit dem ehemaligen Partner, geben aber an, keine Beziehung zu führen $\left.{ }^{* *}\right) 12$ Fälle führen einen gemeinsamen Haushalt mit dem Ehepartner, geben aber an, keine Beziehung zu führen

Quelle: pairfam/DemoDiff Welle 1

Um zu einer Kategorisierung von Lebensformen zu gelangen, haben wir die in Abbildung 1 dargestellten Kategorien in vier Lebensformentypen gruppiert. Die Gruppierung lehnt sich an bisherige Klassifikationsversuche an, in denen die Partnerschaftsform und der Haushaltskontext die dominierenden Elemente bei der Gruppierung von Lebensformen darstellen (Huinink und Konietzka 2007). Da die eheliche Familie jedoch weiterhin ein Bezugspunkt der sozialpolitischen Regelungen ist, bildet die eheliche Lebensgemeinschaft eine übergeordnete Kategorie im Lebensformenschema: 
- Personen in ehelichen Lebensgemeinschaften umfassen jene, die verheiratet sind und mit dem Ehepartner in einer partnerschaftlichen Beziehung leben. Auch Personen, die nicht mit dem Ehepartner zusammen wohnen, werden als Personen in ehelicher Lebensgemeinschaft definiert, sofern sie eine partnerschaftliche Beziehung zum Ehepartner bzw. zur Ehepartnerin pflegen. Auf Grund des Scheidungsprozesses, der sich über mehrere Jahre hinstrecken kann, ist es prinzipiell möglich, dass sich eine verheiratete Person in einer Partnerschaft mit einem neuen Partner befindet, mit dem sie möglicherweise bereits zusammen lebt (siehe Abbildung 1). Diese Personen werden nicht als eheliche Lebensgemeinschaften betrachtet.

- Personen in nichtehelichen Lebensgemeinschaften (NEL) umfassen jene, die mit einem Partner oder einer Partnerin im selben Haushalt leben, mit dem bzw. der sie nicht verheiratet sind. Dies bedeutet, dass auch geschiedene und verwitwete Personen unter diese Kategorie gefasst werden, wenn sie mit einem neuen Partner bzw. einer neuen Partnerin zusammenleben. Verheiratete werden ebenfalls als nichteheliche Lebensgemeinschaft gefasst, wenn sie sich von dem Ehepartner bzw. von der Ehepartnerin getrennt haben und mit einem neuen Partner bzw. einer neuen Partnerin zusammenleben.

- $\quad$ Personen in Living-Apart-Together-Beziehungen (LAT) führen eine nichteheliche Partnerschaft, wobei der Lebenspartner bzw. die Lebenspartnerin nicht im gleichen Haushalt lebt. Auch geschiedene und verwitwete Personen können unter diese Kategorie gefasst werden, wenn sie eine Partnerschaft mit getrennten Haushalten angegeben haben. Verheiratete Personen werden als LAT klassifiziert, wenn sie sich von dem Ehepartner bzw. der Ehepartnerin getrennt haben und eine neue Partnerschaft mit getrennten Haushalten eingegangen sind. 
- Partnerlose sind Personen, die, unabhängig vom Familienstand, zum Befragungszeitpunkt keinen Partner bzw. keine Partnerin haben.

Tabelle 1 zeigt, in welchen Lebensformen Männer und Frauen der Geburtskohorten 197173 zum Befragungszeitpunkt, d.h. im Alter von 35 bis 39 Jahren, lebten. Die Tabelle unterscheidet nach dem Geschlecht der Befragten sowie nach Ost- und Westdeutschland, wobei sich die Definition von Ost- und Westdeutschland auf die Wohnregion zum Befragungszeitpunkt bezieht. Wie aus der Tabelle zu ersehen ist, ist die eheliche Lebensgemeinschaft vor allem in Westdeutschland die dominante Lebensform, während in Ostdeutschland die nichteheliche Lebensgemeinschaft stärker verbreitet ist. Betrachtet man die Unterschiede zwischen Männern und Frauen, fällt zum einen der höhere Anteil Unverheirateter unter den männlichen Befragten auf, der sich insbesondere durch das höhere Erstheiratsalter von Männern erklären lässt.

Tabelle 1: Lebensformen der Geburtskohorten 1971-73 zum Befragungszeitpunkt, Spaltenprozente

\begin{tabular}{|l|cc|cc|}
\hline & \multicolumn{2}{|c|}{ Frauen } & \multicolumn{2}{c|}{ Männer } \\
& Ostdeutsch- & Westdeutsch- & Ostdeutsch- & Westdeutsch- \\
& land & land & land & land \\
\hline Partnerlos & 17,9 & 17,3 & 21,4 & 22,1 \\
LAT & 7,1 & 6,6 & 10,3 & 6,6 \\
NEL & 21,3 & 11,7 & 26,5 & 16,2 \\
Ehe & 53,7 & 64,5 & 41,8 & 55,1 \\
\hline Fallzahlen & 744 & 1.768 & 690 & 1.445 \\
\hline
\end{tabular}

Quelle: pairfam/DemoDiff Welle 1 (gewichtete Werte) 


\subsection{Elternschaft und Lebensform in verschiedenen Altersphasen}

Für eine umfassende Darstellung familialer Lebensformen ist es notwendig neben der Lebensform auch nach dem Elternschaftsstatus zu unterscheiden. Prinzipiell wird eine Unterscheidung zwischen biologischer Elternschaft sowie Adoptiv-, Pflege- und Stiefelternschaft getroffen (Vaskovics 2009; Schwab und Vaskovics 2011). Folgt man der Logik einer haushaltsübergreifenden Sichtweise, müsste zudem berücksichtigt werden, ob eine Koresidenz eines Kindes mit dem befragten Elternteil vorliegt. Da Befragte mehr als ein Kind haben können, ergeben sich eine Vielzahl von Elternschafts- und Koresidenzkonstellationen. Um die Darstellung der Familienformen übersichtlich zu gestalten, lassen wir im Folgenden den Aspekt der Koresidenz der Kinder und der Stiefelternschaft unberücksichtigt (siehe hierzu Steinbach 2008; Kreyenfeld und Martin 2011; Feldhaus und Huinink 2011). Unberücksichtigt bleibt auch die Dimension der Pflege- und Adoptivelternschaft (siehe hierzu Alt und Lange 2011). Unterscheidungsdimension für die Klassifikation von Lebensformen ist im Folgenden nur das Vorliegen einer biologischen Elternschaft, unabhängig von der Koresidenz des Kindes bzw. der Kinder. Der Nachteil dieser Klassifikation ist, dass dem Vorliegen einer biologischen Elternschaft das Primat eingeräumt wird, es jedoch für den alltäglichen Lebenszusammenhang möglicherweise relevanter ist, ob ein Kind bzw. Kinder im Haushalt leben. Wir haben dennoch dieses Vorgehen gewählt, da die weiteren Analysen die Dynamik der Lebensformen nach der Familiengründung fokussieren und somit die Geburt des ersten Kindes als einschneidendes Lebensereignis definiert wird.

Tabelle 2 gibt für Personen mit Kindern die jeweilige Lebensform wieder. Da Lebensformen über den Lebenslauf variieren, sind sie nach Alter dargestellt. Zudem gibt die Tabelle für jedes Alter den Anteil an Befragten mit Kindern an. Ostdeutsche Frauen der Geburtskohorten 1971-73 sind demnach deutlich früher in ihren Lebensläufen Mutter 
geworden als Westdeutsche. Mit 30 Jahren haben bereits 65 Prozent der ostdeutschen Frauen mindestens ein Kind bekommen, in Westdeutschland sind es hingegen nur 53 Prozent der Frauen. Bis zum Alter 35 schwächen sich die Unterschiede in den Anteilen Kinderloser ab, da mehr west- als ostdeutsche Frauen in dieser Lebensphase eine Familie gründen. Unter ost- und westdeutschen Männern ist Kinderlosigkeit ähnlich verbreitet. Der Anteil an Vätern steigt in beiden Landesteilen gleichermaßen mit dem Alter.

Über den Lebenslauf hinweg zeigt sich insgesamt eine deutliche Konstanz der Verteilung der Lebensformen innerhalb der betrachteten Gruppen. Die Ehe ist in jeder Altersgruppe die am häufigsten gewählte Lebensform von Müttern und Vätern. Eine Ausnahme stellen lediglich Ostdeutsche dar, die im Alter von 20 Mutter oder Vater geworden sind. Aufgrund geringer Fallzahlen können zu den Lebensformenvariationen in diesem Alter jedoch keine gesicherten Aussagen gemacht werden. Mit zunehmendem Lebensalter wächst in Ostdeutschland der Anteil an ehelichen Lebensgemeinschaften unter den Personen mit Kindern. Auffällig ist dennoch, dass auch im späteren Alter die nichteheliche Lebensgemeinschaft in Ostdeutschland deutlich häufiger verbreitet ist als in Westdeutschland. Im Alter von 35 Jahren sind es 24 Prozent der ostdeutschen Mütter und 29 Prozent der Väter, die in dieser Lebensform zu finden sind. In Westdeutschland sind es gerade mal 11 Prozent der Mütter und Väter. LAT-Beziehungen haben unter den älteren Befragten mit Kindern weder in Ost- noch in Westdeutschland eine Bedeutung. Diese Lebensform ist in erster Linie unter den jungen Müttern und Vätern in Ostdeutschland zu finden. 
Table 2: Lebensformen von Frauen und Männern mit Kindern der Geburtskohorten 197173, Spaltenprozente

\begin{tabular}{|c|c|c|c|c|}
\hline Mütter Ostdeutschland & Alter 20 & Alter 25 & Alter 30 & Alter 35 \\
\hline Partnerlos & 13,9 & 12,8 & 12,6 & 12,1 \\
\hline LAT & 20,7 & 8,5 & 6,7 & 6,4 \\
\hline NEL & 41,3 & 29,4 & 27,2 & 24,1 \\
\hline Ehe & 24,2 & 49,3 & 53,5 & 57,5 \\
\hline Fallzahlen & 97 & 256 & 456 & 538 \\
\hline \% Mütter nach Alter & 12,5 & 35,8 & 64,5 & 79,6 \\
\hline Mütter Westdeutschland & Alter 20 & Alter 25 & Alter 30 & Alter 35 \\
\hline Partnerlos & 17,3 & 11,1 & 10,5 & 10,4 \\
\hline LAT & 6,1 & 5,0 & 5,0 & 4,8 \\
\hline NEL & 16,5 & 14,9 & 12,1 & 10,2 \\
\hline Ehe & 60,1 & 69,0 & 72,4 & 74,6 \\
\hline Fallzahlen & 62 & 306 & 687 & 975 \\
\hline$\%$ Mütter nach Alter & 6,7 & 27,6 & 53,2 & 74,2 \\
\hline Väter Ostdeutschland & Alter 20 & Alter 25 & Alter 30 & Alter 35 \\
\hline Partnerlos & 12,8 & 13,4 & 12,7 & 10,9 \\
\hline LAT & 22,5 & 11,4 & 4,9 & 6,4 \\
\hline NEL & 38,9 & 36,0 & 38,3 & 29,3 \\
\hline Ehe & 25,8 & 39,1 & 44,1 & 53,4 \\
\hline Fallzahlen & 15 & 106 & 275 & 389 \\
\hline \% Väter nach Alter & 2,0 & 14,8 & 39,9 & 59,9 \\
\hline Väter Westdeutschland & Alter 20 & Alter 25 & Alter 30 & Alter 35 \\
\hline Partnerlos & 3,9 & 8,9 & 8,1 & 6,0 \\
\hline LAT & 19,8 & 5,2 & 3,2 & 3,3 \\
\hline NEL & 11,1 & 15,5 & 16,3 & 10,9 \\
\hline Ehe & 65,3 & 70,4 & 72,3 & 79,9 \\
\hline Fallzahlen & 17 & 117 & 356 & 587 \\
\hline \% Väter nach Alter & 2,1 & 13,2 & 34,3 & 53,1 \\
\hline
\end{tabular}

Quelle: pairfam/DemoDiff Welle 1 (gewichtete Werte) 


\section{Lebensformendynamik der Frauen der Geburtskohorten 1971-1973}

\subsection{Kopplung von Heirat und Familiengründung}

Abbildung 2 gibt einen Einblick in die Kopplung von Lebensform und Fertilitätsbiographie. Dargestellt ist die Lebensform zum Zeitpunkt der Geburt des ersten Kindes, neun Monate vor der Geburt und zum Zeitpunkt der Geburt des zweiten Kindes. Diese und die folgenden Darstellungen haben wir auf weibliche Befragte begrenzt. Der wesentliche Grund für dieses Vorgehen ist, dass in den Analysen nur Personen berücksichtigt werden können, die bereits Elternschaftserfahrung gemacht haben. Die befragten Frauen in unserer Stichprobe haben dies zu einem größeren Anteil getan als dies für die männlichen Befragten der Fall ist, sodass die Stichprobe der weiblichen Personen mit Kindern weniger selektiv ist als die der männlichen Population.

Abbildung 2 zeigt, dass zwei Drittel der westdeutschen, aber nur gut ein Drittel der ostdeutschen Frauen zum Zeitpunkt der Geburt ihres ersten Kindes verheiratet sind. In Ostdeutschland lebt ein erheblicher Anteil der Mütter zum Zeitpunkt der Erstgeburt in einer nichtehelichen Lebensgemeinschaft (43 Prozent). Partnerlosigkeit bei Geburt des ersten Kindes ist zwar eher die Ausnahme, jedoch sind es immerhin 12 Prozent der ostdeutschen und acht Prozent der westdeutschen Frauen, die keinen Partner haben, wenn ihr erstes Kind geboren wird. Ost-West-Unterschiede existieren auch in der Frage, ob eine Schwangerschaft einen Anlass zur Heirat darstellt. In Westdeutschland zeigt sich, dass gerade zwischen Schwangerschaftsbeginn und Geburt des ersten Kindes der Anteil der unverheirateten Frauen stark zurückgeht (von 54 auf 34 Prozent). Die Schwangerschaft stellt damit im Westen immer noch einen wesentlichen Anlass zur Eheschließung dar. In Ostdeutschland geht der Anteil unverheirateter Frauen zwischen diesen beiden Zeitpunkten nur mäßig (von 75 auf 63 Prozent) zurück. Betrachtet man den Zeitpunkt der Geburt des 
zweiten Kindes, stellt man für Ost- wie auch für Westdeutschland fest, dass die weite Mehrzahl der Frauen verheiratet ist. Inwiefern dies daran liegt, dass Personen zwischen der Geburt des ersten und zweiten Kindes heiraten oder es in erster Linie die verheirateten Frauen sind, die ein zweites Kind bekommen, kann jedoch auf Basis dieser Abbildung nicht beurteilt werden.

Abbildung 2a: Lebensformen und Kindgeburt, Frauen der Geburtskohorten 1971-73, Ostdeutschland

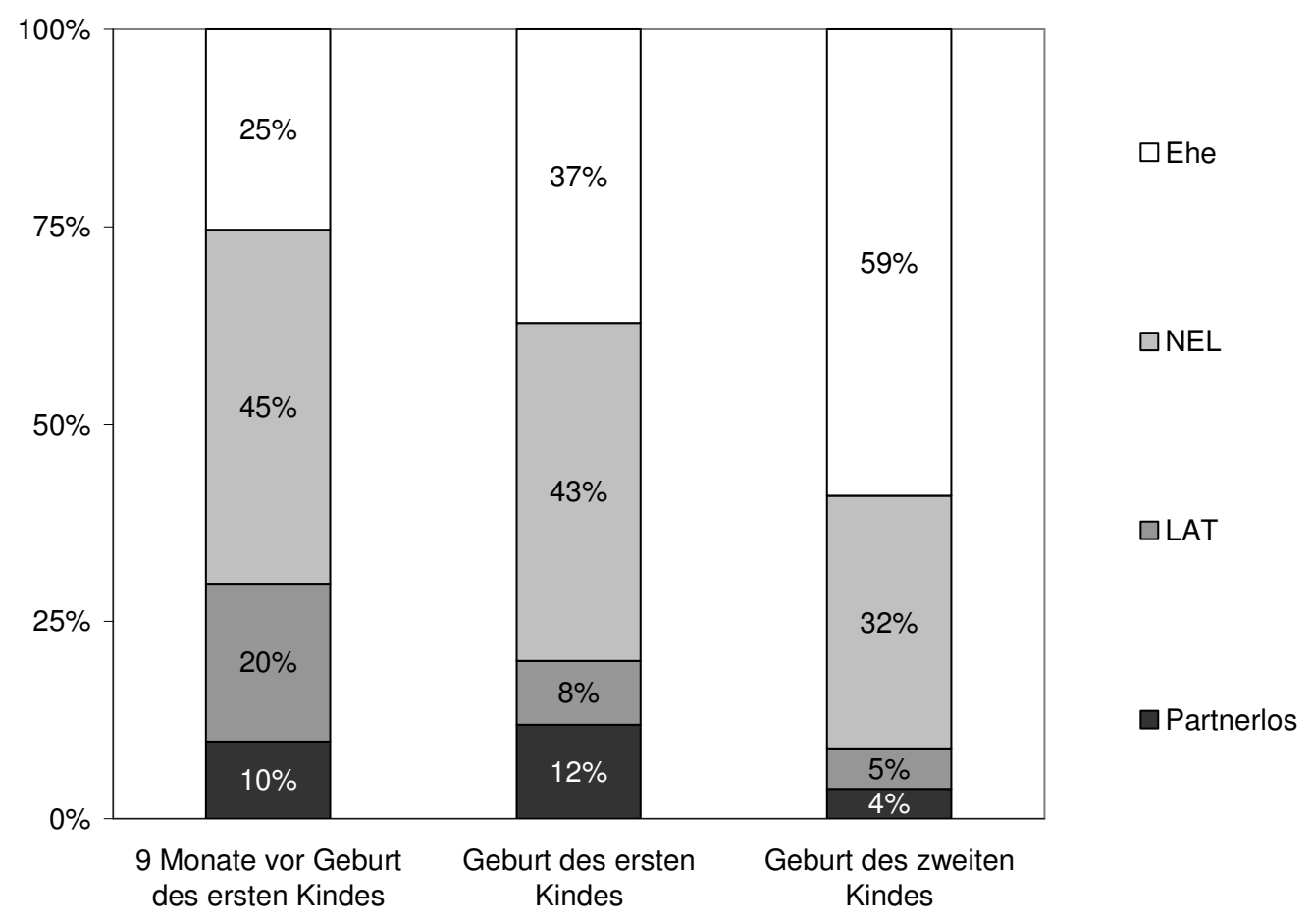


Abbildung 2b: Lebensformen und Kindgeburt, Frauen der Geburtskohorten 1971-73, Westdeutschland

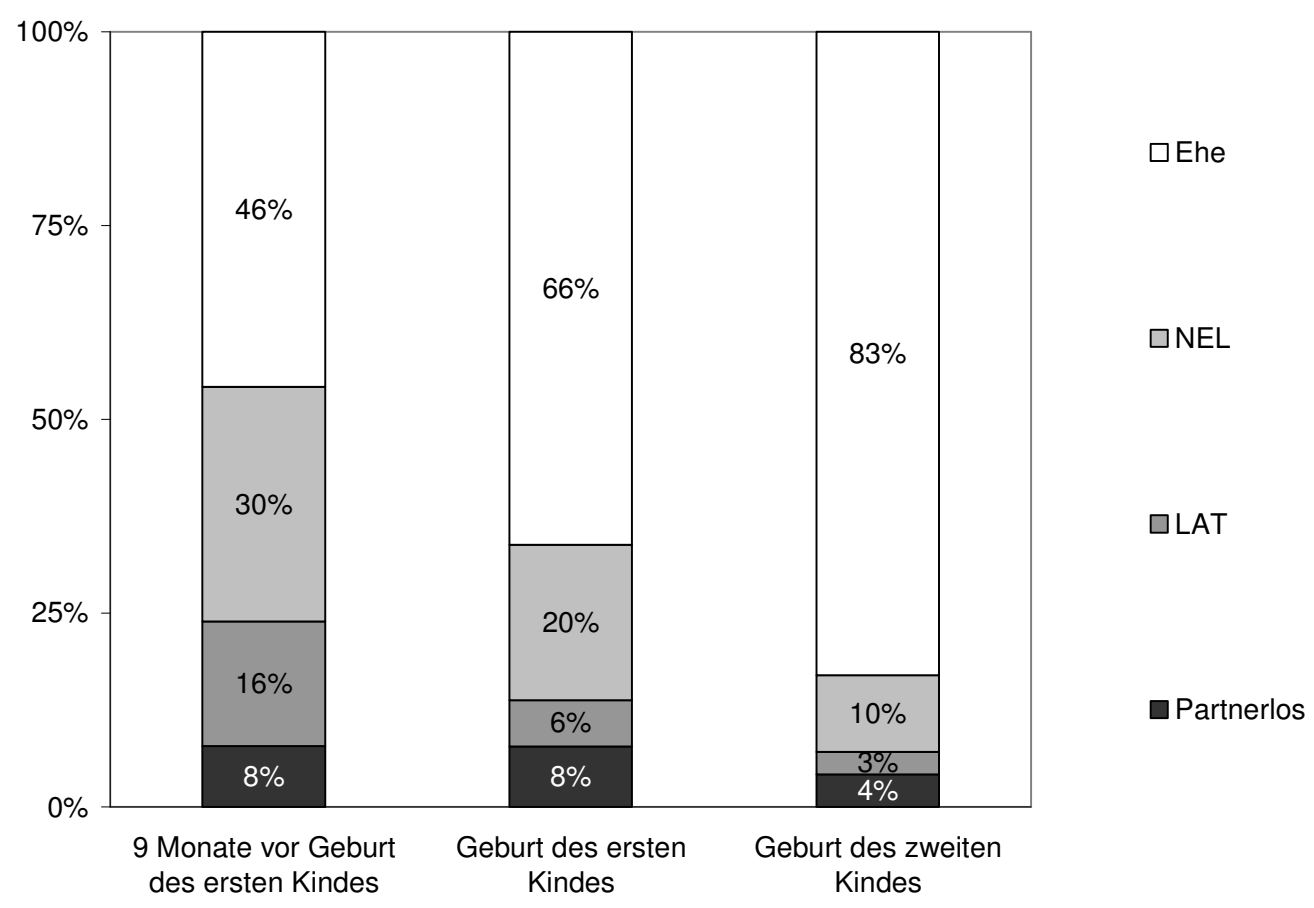

Quelle: pairfam/DemoDiff Welle 1 (gewichtete Werte)

\subsection{Nichteheliche Lebensgemeinschaft mit Kindern: Eine stabile Lebensform?}

In Ostdeutschland leben 43 Prozent aller Frauen in einer nichtehelichen Lebensgemeinschaft (NEL), wenn das erste Kind geboren wird. In Westdeutschland heiratet die Mehrzahl vor der Familiengründung. Dennoch sind es auch in Westdeutschland 20 Prozent, die zum Zeitpunkt der Erstgeburt in einer NEL leben. Eine wesentliche Frage ist, ob nichteheliche Lebensgemeinschaften eine stabile Lebensform darstellen, oder auch nach der Familiengründung Eheschließungen stattfinden. In der Literatur wird häufig die Vorstellung vertreten, dass nichteheliche Lebensgemeinschaften eher temporärer Natur sind, da sie entweder zügig in eheliche Lebensgemeinschaften transferiert werden oder sich die Partnerschaften auflösen (Klein und Lauterbach 1999). 
Abbildung 3 zeigt vor dem Hintergrund dieser Fragestellung die Stabilität von nichtehelichen Lebensgemeinschaften. Die Abbildung enthält Personen, die bei der Geburt des ersten Kindes in einer nichtehelichen Lebensgemeinschaft lebten. Dargestellt ist der Zeitraum ab Geburt bis zum achten Geburtstag des ersten Kindes. Da der Übergang aus der NEL in eine Ehe erfolgen kann, die Partner sich jedoch auch trennen oder lediglich in verschiedene Haushalte ziehen können (LAT), sind verschiedene Folgezustände möglich. Um den Übergang in diese unterschiedlichen Folgezustände abzubilden, haben wir kumulierte Inzidenzfunktionen geschätzt (Gooley et al. 1999).

Die Abbildung gibt Hinweise auf eine gewisse Stabilität der NEL in den ersten Familienjahren. In Ostdeutschland leben 30 Prozent der Frauen, die bei Geburt des ersten Kindes in einer NEL lebten auch noch in dieser Lebensform, wenn das erste Kind acht Jahre alt ist. In Westdeutschland ist der Anteil mit 15 Prozent deutlich geringer. Gerade in Westdeutschland heiraten noch relativ viele Frauen nach der Familiengründung. Dies gilt insbesondere für die unmittelbare Zeit nach der Geburt des ersten Kindes, doch auch zwischen dem zweiten und fünften Lebensjahr sind in Westdeutschland anteilmäßig mehr Eheschließungen zu verzeichnen als in Ostdeutschland. Direkte Partnerschaftstrennungen aus der NEL sind sowohl in Ost- als auch in Westdeutschland nur sehr selten in den ersten zwei Lebensjahren des Kindes zu finden. 
Abbildung 3a: Wechsel von der NEL in andere Lebensformen nach Alter des ersten Kindes (in Monaten), Frauen der Kohorten 1971-73, Ostdeutschland

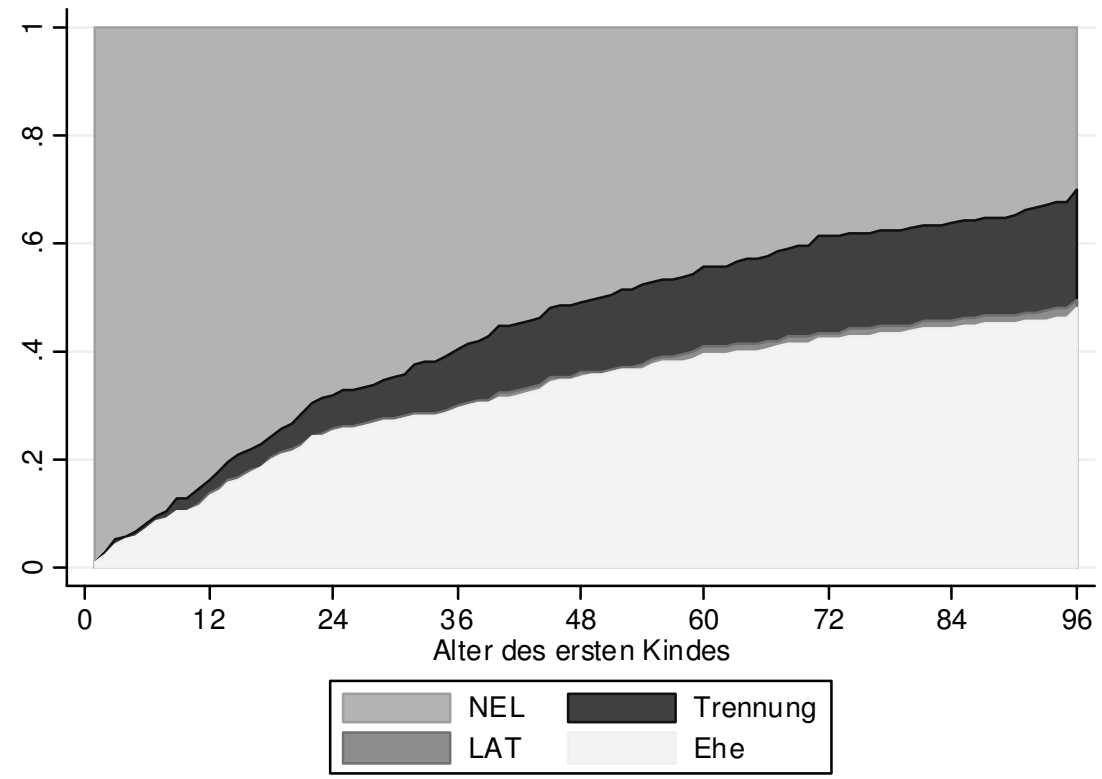

Abbildung 3b: Wechsel von der NEL in andere Lebensformen nach Alter des ersten Kindes (in Monaten), Frauen der Kohorten 1971-73, Westdeutschland

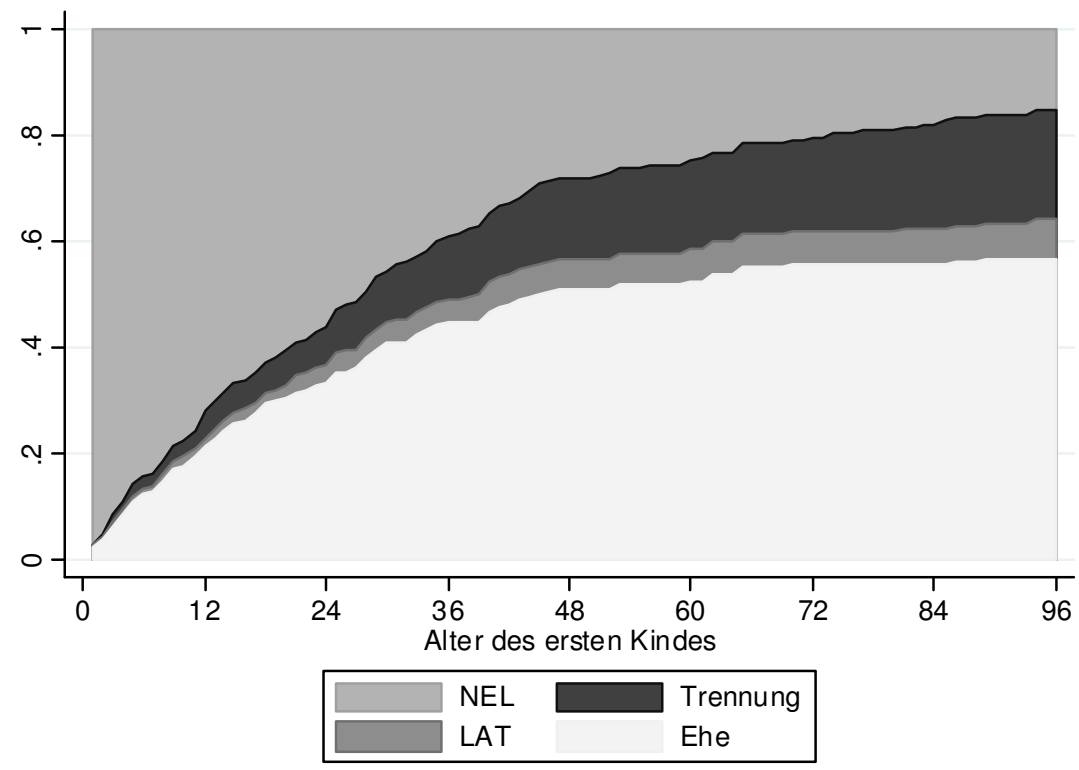

Anmerkung: Die Abbildungen basieren auf kumulierten Inzidenzfunktionen.

Quelle: pairfam/DemoDiff Welle 1 (ungewichtete Analysen) 


\subsection{Trennung nach der Familiengründung}

Trennung und Scheidung sind einschneidende Ereignisse in den Lebensläufen von Personen. Dies gilt umso mehr, wenn aus einer Partnerschaft Kinder hervorgegangen sind. Die bisherigen Analysen zur Dynamik von nichtehelichen Lebensgemeinschaften geben bereits Hinweise auf das Trennungsrisiko von nichtehelichen Lebensgemeinschaften. Eine allgemeine Schlussfolgerung auf die Stabilität von Paarbeziehungen nach der Familiengründung ist hiermit jedoch nicht möglich, da lediglich der erste Übergang aus der nichtehelichen Lebensgemeinschaft in diesen Analysen erfasst wird. Es wird nicht berücksichtigt, dass eine Trennung erfolgen kann, nachdem eine NEL in eine Ehe transformiert wurde. Zudem ist die Betrachtung nur auf Partnerschaften beschränkt, die innerhalb einer nichtehelichen Lebensgemeinschaft eine Familie gegründet haben. Diese sind jedoch in Ost- und Westdeutschland unterschiedlich verbreitet.

Abbildung 4 gibt nun Aufschluss über das Trennungsverhalten von allen Frauen, nachdem sie ihr erstes Kind geboren haben. In die Darstellung gehen auch Frauen ein, die sich bereits zum Zeitpunkt der Geburt getrennt haben oder nie eine partnerschaftliche Beziehung zu dem Vater des Kindes pflegten. ${ }^{4}$ Im betrachteten Zeitraum ist der Anteil an getrennten Müttern in Ostdeutschland durchgängig höher als in Westdeutschland. Dies ist hauptsächlich auf den höheren Anteil an bei Geburt partnerlosen Frauen in Ostdeutschland und der höheren Partnerschaftsinstabilität von ostdeutschen Müttern im ersten Lebensjahr ihres Kindes zurückzuführen. Wie aus der Abbildung zu ersehen ist, sind zum Zeitpunkt der Geburt des ersten Kindes bereits elf Prozent der Frauen in Ostdeutschland und sieben

4 Die Prozesszeit ist die Zeit seit Geburt des ersten Kindes bis zur Trennung vom Partner bzw. von der Partnerin, den bzw. die die Befragte zu dem Zeitpunkt hatte als sie ihr erstes Kind geboren hat. Vor dem Hintergrund, dass einer außerpartnerschaftlichen Schwangerschaft i.d.R. eine sexuelle Beziehung vorausgegangen ist, werden auch Personen berücksichtigt, die keine Partnerschaft angegeben haben. 
Prozent der westdeutschen Frauen getrennt. ${ }^{5}$ Acht Jahre nach der ersten Kindgeburt sind 27 Prozent der westdeutschen Frauen nicht mehr mit ihrem Partner zusammen, im Osten haben bereits 34 Prozent eine Trennung hinter sich. Demzufolge ist insbesondere aufgrund der unterschiedlichen Ausgangslage die Trennungswahrscheinlichkeit für Personen mit Kindern in Ostdeutschland deutlich höher als in Westdeutschland.

Abbildung 4: Anteil der Mütter der Kohorten 1971-73, die sich getrennt haben, nach Alter des ersten Kindes

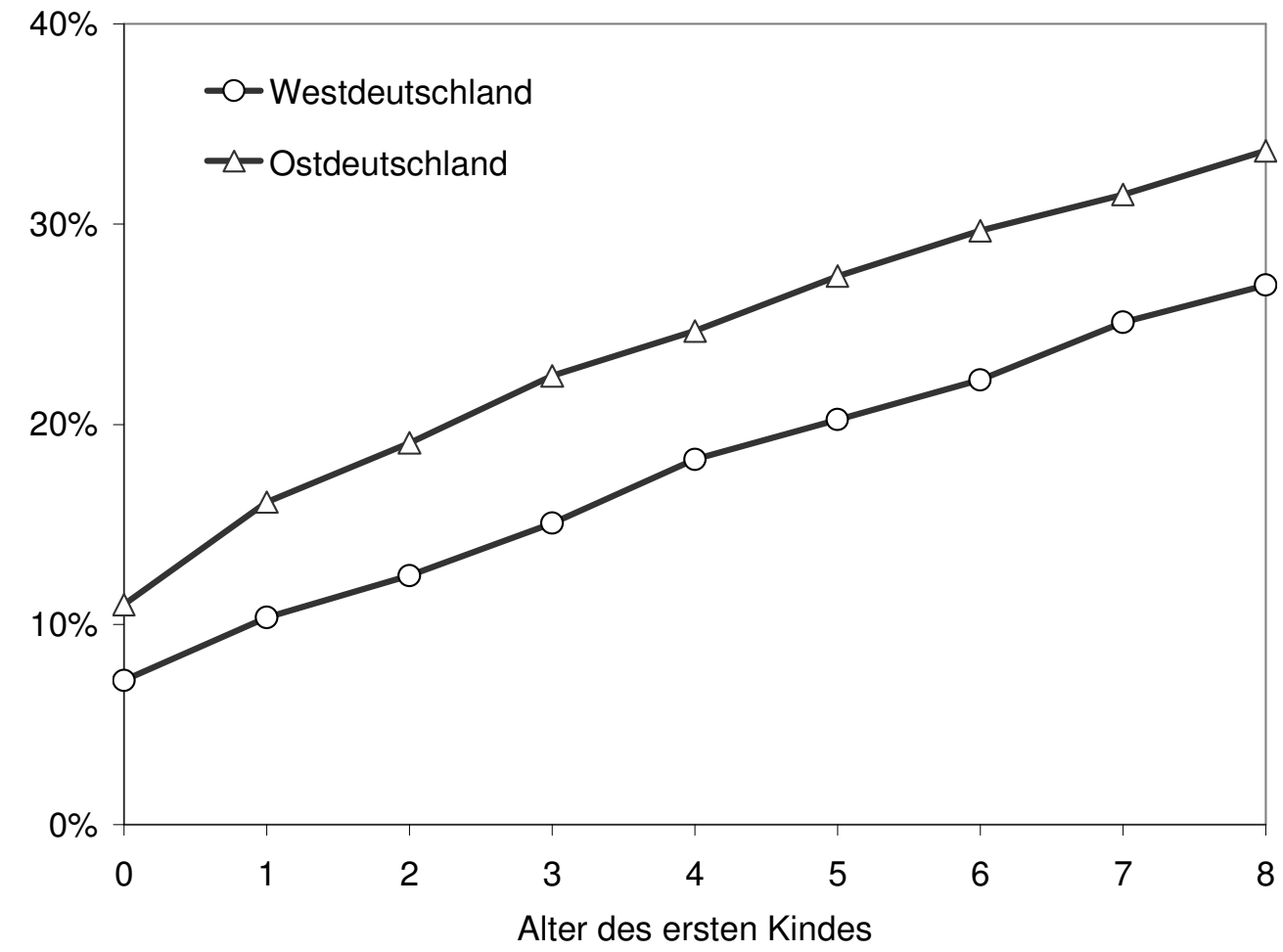

Anmerkung: Die Abbildungen basieren auf Kaplan-Meier-Failure-Funktionen

Quelle: pairfam/DemoDiff Welle 1 (ungewichtete Analysen) da die Überlebensfunktionen nicht gewichtet wurden. 
Abbildung 5 unterscheidet die Trennungswahrscheinlichkeit nach der Form der Partnerschaft zum Zeitpunkt der Kindgeburt. Ausgeschlossen von dieser Darstellung werden Mütter, die sich zum Zeipunkt der Geburt keine Partnerschaft angegeben haben. Zudem ist die Trennungswahrscheinlichkeit von Frauen nicht dargestellt, die ihr erstes Kind in einer LAT-Beziehung bekommen haben, da diese Gruppe relativ klein ist (siehe Abbildung 2). Entsprechend unterscheidet Abbildung 5 nur Frauen, die entweder in einer nichtehelichen Lebensgemeinschaft oder in einer ehelichen Lebensgemeinschaft lebten, als das erste Kind geboren wurde. Die Abbildung zeigt, dass Mütter, die mit ihrem Partner zum Zeitpunkt der Geburt in einer nichtehelichen Lebensgemeinschaft lebten, einem höheren Trennungsrisiko ausgesetzt sind als Mütter, die sich zum gleichen Zeitpunkt in einer ehelichen Lebensgemeinschaft befanden. Auffällig sind zudem die Ost-WestUnterschiede. Unter den ehelichen Lebensgemeinschaften sind die ostdeutschen Partnerschaften instabiler, während es bei den nichtehelichen Lebensgemeinschaften die westdeutschen Partnerschaften sind, die eine höhere Instabilität aufweisen. Erklären lässt sich dieser Sachverhalt am ehesten mit der Tatsache, dass die westdeutsche Population, die bei Geburt des ersten Kindes (noch) unverheiratet ist, deutlich selektiver ist als die Population in Ostdeutschland, wo die nichteheliche Familiengründung wesentlich verbreiteter ist. 
Abbildung 5a: Anteil der Mütter der Kohorten 1971-73, die sich getrennt haben, nach Alter des ersten Kindes; Frauen, die bei Geburt des ersten Kindes in einer NEL lebten

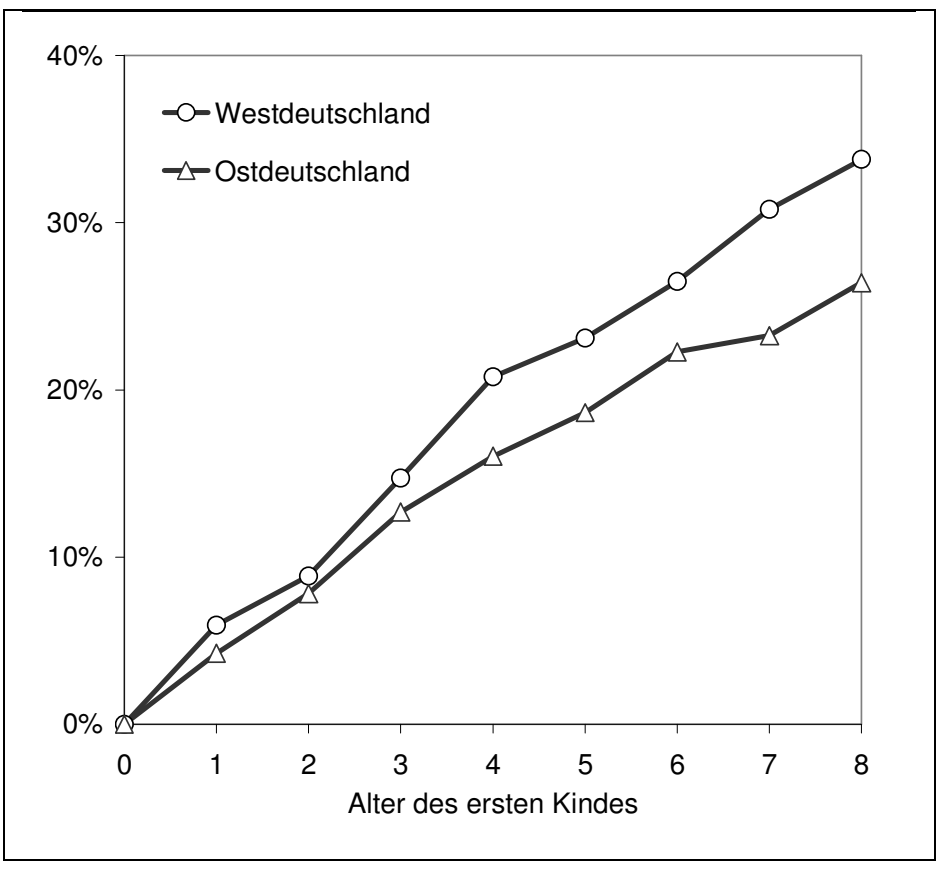

Abbildung 5b: Anteil der Mütter der Kohorten 1971-73, die sich getrennt haben, nach Alter des ersten Kindes; Frauen, die bei Geburt des ersten Kindes in einer Ehe lebten

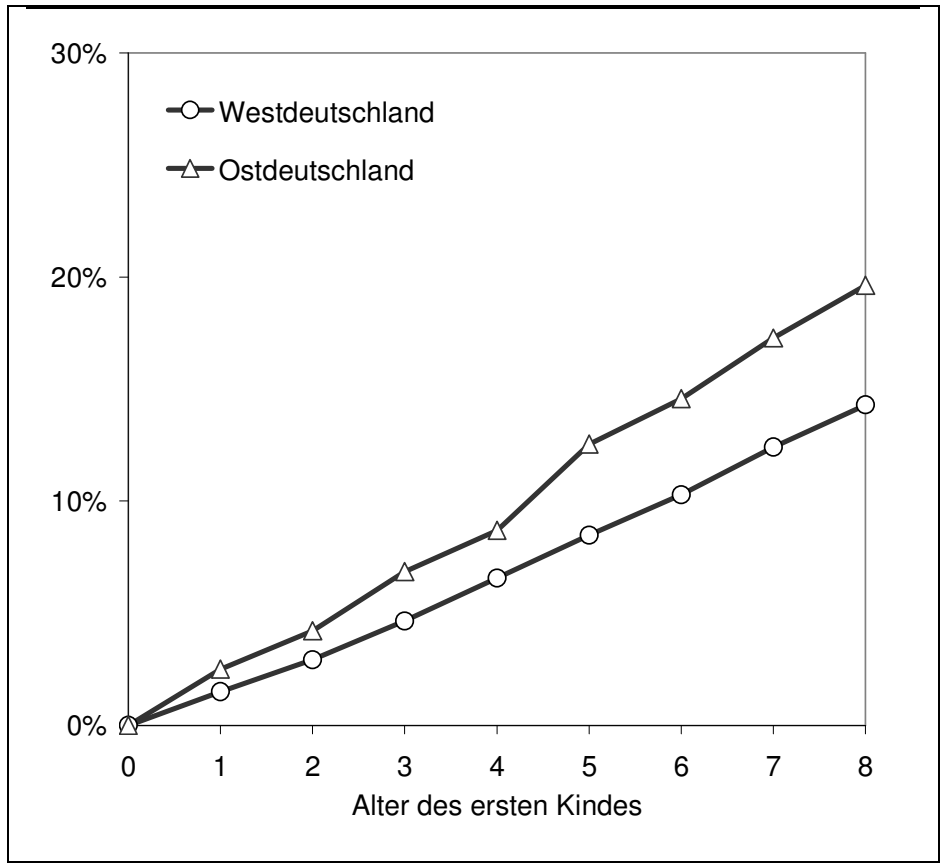

Anmerkung: Die Abbildungen basieren auf Kaplan-Meier-Failure-Funktionen Quelle: pairfam/DemoDiff Welle 1 (ungewichtete Analysen) 


\section{Fazit}

Ziel dieses Beitrags war es die Diversität der Lebens- und Familienformen aus einer Längsschnittperspektive zu betrachten. Als Datensatz haben wir das Beziehungs- und Familienpanel verwendet, wobei wir uns auf die Geburtskohorten 1971-1973 beschränkt haben, die zum Befragungszeitpunkt wesentliche familiale Übergänge, wie die Geburt des ersten Kindes, weitgehend realisiert hatten. Obwohl diese Geburtsjahrgänge ihre Partnerschafts- und Elternschaftsentscheidungen im wiedervereinigten Deutschland getroffen haben, bestehen für diese Kohorten in den Lebens- und Familienformen erhebliche Ost-West-Unterschiede. Im Einklang mit bisherigen Studien zeigt sich die relativ hohe Verbreitung nichtehelicher Lebensgemeinschaften mit Kindern in Ostdeutschland, wobei diese Lebensform vor allem im frühen Lebenslauf eine besondere Relevanz besitzt. Frühe Erstelternschaft ist insbesondere in Ostdeutschland mehrheitlich nichteheliche Elternschaft. In Westdeutschland dominiert in jedem Lebensalter die eheliche Lebensgemeinschaft unter den Personen mit Kind.

Eine Darstellung der Lebensformen zum Zeitpunkt der Geburt des ersten Kindes unterstreicht zwar vorhergehende Befunde, die darauf verweisen, dass der Übergang in die Ehe in Westdeutschland mehrheitlich vor der Familiengründung realisiert wird. Auf immerhin ein Drittel der westdeutschen Frauen der Kohorten 1971-73 trifft dies jedoch nicht zu. In Ostdeutschland sind es sogar deutlich mehr als die Hälfte der Frauen, die bei Erstgeburt nicht verheiratet sind. In beiden Landesteilen leben Frauen, die bei Geburt ihres ersten Kindes unverheiratet sind, mehrheitlich in einer nichtehelichen Lebensgemeinschaft.

Die weitere dynamische Betrachtung zeigt, dass auch nach der Familiengründung Lebensformen einer erheblichen Dynamik unterworfen sind. Westdeutsche Frauen, die in einer NEL ihr erstes Kind bekommen haben, heiraten häufig noch in den ersten Jahren nach Geburt des ersten Kindes. In Ostdeutschland ist die Wahrscheinlichkeit geringer nach 
der Erstgeburt noch zu heiraten. Dennoch hat etwa die Hälfte der ostdeutschen Frauen, die bei Geburt des ersten Kindes in einer NEL lebten, acht Jahre später geheiratet. Dieser Befund deutet darauf hin, dass es neben der Familiengründung weitere Determinanten gibt, welche für eine Eheschließung relevant sind.

Betrachtet man die Trennungsverhalten nach Familiengründung zeigt sich, dass die Trennungswahrscheinlichkeit ostdeutscher Mütter höher als die westdeutscher Frauen mit Kindern ist. Wenn das erste Kind acht Jahre alt ist, haben sich 27 Prozent der Frauen in Westdeutschland und 34 Prozent der ostdeutschen Frauen von dem Partner, den sie bei Geburt hatten, getrennt. Für Ostdeutschland fällt dabei der relativ hohe Anteil der Frauen auf, der bereits bei Geburt des ersten Kindes keinen Partner (mehr) hatte. Dieser Aspekt ist vor allem vor dem Hintergrund relevant, dass die Kinder dieser Befragten bereits im frühen Alter die Erfahrung machen in einem Einelternhaushalt zu leben und möglicherweise früh in ihren Lebensläufen den Eintritt in eine Stieffamilie erfahren. Im Hinblick auf Ost-WestUnterschiede ist an dieser Stelle ferner relevant, dass die Trennungswahrscheinlichkeit von westdeutschen Müttern, die bei Geburt ihres ersten Kindes in einer NEL leben, höher ist als von vergleichbaren ostdeutschen Frauen. Dies weist auf eine unterschiedliche Selektivität der nichtehelichen Lebensgemeinschaft in den beiden Teilen Deutschlands hin. Insgesamt zeigt die Längsschnittbetrachtung, dass auch nach der Familiengründung Lebensformen einer erheblichen Dynamik unterliegen, welche sich zudem unterschiedlich zwischen Ost- und Westdeutschland darstellt. Dieser Befund ist vor allem vor dem Hintergrund relevant, dass die Analyse von Lebens- und Familienformen zumeist einer Querschnittslogik folgt. In diesem Forschungsbereich sind in der Vergangenheit vor allem Fortschritte erzielt worden, indem die haushaltsübergreifenden Strukturen, in denen Befragte leben, erfasst wurden. Die Komplexität, die Lebensformen über den Lebenslauf entfalten, ist bislang hingegen nicht in gleicher Weise Rechnung getragen worden. Gerade 
für die jungen Kohorten, deren Lebensläufe auch nach der Familiengründung noch deutlichen Dynamiken unterworfen sind, ist es umso relevanter geworden, eine Längsschnittperspektive einzunehmen, um deren familiale Lebenssituation verstehen zu können. 


\section{Referenzen}

Alt, Christian /Lange, Andreas (2011): Kindschaftskonstellationen in Vater-MutterFamilien und in Einelternfamilien. In: Schwab, Dieter/ Vaskovics, Laszlo A. (Hrsg.): Pluralisierung der Elternschaft und Kindschaft. Zeitschrift für Familienforschung Sonderheft 8, Leverkusen: Verlag Barbara Budrich.

Brüderl, Josef (2004): Die Pluralisierung partnerschaftlicher Lebensformen in Westdeutschland und Europa. Aus Politik und Zeitgeschichte B19: 3-10.

Elzinga, Cees /Liefbroer, Aart (2007): De-standardization of family-life trajectories of young adults: a cross-national comparison using sequence analysis. European Journal of Population 23: 225-250.

Feldhaus, Michael /Schlegel, Monika (2011): Vielfalt (mobiler) Lebensformen? Aus Politik und Zeitgeschichte B41: 37-38.

Feldhaus, Michael /Huinink, Johannes (2011): Multiple Elternschaft. Eine Analyse zur Vielfalt von Elternschaft in Folgepartnerschaften. Zeitschrift für Familienforschung (im Erscheinen).

Fokkema, Tineke /Liefbroer, Aart (2008): Trends in living arrangements in Europe: Convergence or divergence? Demographic Research 19: 1351-1418.

Gooley, Ted A. /Leisenring, Wendy /Crowley, John /Storer, Barry. E. (1999): Estimation of failure probabilities in the presence of competing risks: new representations of old estimators. Statistics in Medicine 18: 695-706.

Huinink, Johannes /Brüderl, Josef, et al. (2011): Panel Analysis of Intimate Relationships and Family Dynamics (pairfam). Zeitschrift für Familienforschung 23: 77-101. 
Huinink, Johannes /Konietzka, Dirk (2007): Familiensoziologie. Eine Einführung. Frankfurt/Main.

Klein, Thomas /Lauterbach, Wolfgang (1999) (Hrsg.): Nichteheliche Lebensgemeinschaften. Analyse zum Wandel partnerschaftlicher Lebensformen. Opladen: Leske+Budrich.

Kreyenfeld, Michaela /Martin, Valerie (2011): Economic conditions of stepfamilies from a cross-national perspective. Zeitschrift für Familienforschung 23: 128-153.

Kreyenfeld, Michaela /Walke, Rainer/Salzburger, Veronika /Schnor, Christine /Bastin, Sonja /Kuhnt, Anne-Kristin (2011): DemoDiff - Wave 1. Supplement to the pairfam-Data Manual. Max Planck Institute for Demographic Research. Technical Report No. 4.

Lesthaeghe, Ron (1992): Der zweite demographische Übergang in den westlichen Ländern: Eine Deutung. Zeitschrift für Bevölkerungswissenschaft 18: 313-354.

Mayer, Karl Ulrich /Eva Schulze (2009): Die Wendegeneration. Lebensverläufe des Jahrgangs 1971. Frankfurt/Main: Campus Verlag.

Nave-Herz, Rosemarie (1997): Pluralisierung familialer Lebensformen - ein Konstrukt der Wissenschaft? In: L. A. Vaskovics (Hrsg.): Familienleitbilder und Familienrealitäten. Opladen: Leske+Budrich: 36-49.

Peuckert, Rüdiger (2008): Familienformen im sozialen Wandel. 7. vollständig überarbeitet Auflage. Opladen: Leske + Budrich.

Schneider, Norbert F. /Hartmann, Kerstin /Limmer, Ruth (2001): Berufsmobilität und Lebensform. Sind berufliche Mobilitätserfordernisse in Zeiten von Globalisierung noch mit Familie vereinbar? Schriftenreihe des Bundesfamilienministeriums, Band 208. Stuttgart: Kohlhammer. 
Schneider, Norbert F. /Rosenkranz, Doris /Limmer, Ruth (1998): Nichtkonventionelle Lebensformen. Entstehung, Entwicklung, Konsequenzen. Opladen: Leske+Budrich.

Schwab, Dieter /Vaskovics, Laszlo A. (Hrsg.) (2011): Pluralisierung der Elternschaft und Kindschaft. Leverkusen: Verlag Barbara Budrich (im Erscheinen).

Statistisches Bundesamt (Destatis) /Wissenschaftszentrum Berlin für Sozialforschung (WZB) /Deutsches Institut für Wirtschaftsforschung (DIW) (2011): Datenreport 2011.

Stauder, Johannes (2011): Regionale Ungleichheit auf dem Partnermarkt? Die makrostrukturellen Rahmenbedingungen der Partnerwahl in regionaler Perspektive. Soziale Welt 62: 41-69.

Steinbach, Anja (2008): Stieffamilien in Deutschland. Zeitschrift für Bevölkerungsforschung 33: 153-180.

Strohmeier, Klaus Peter (1993): Pluralisierung und Polarisierung der Lebensformen in Deutschland. Aus Politik und Zeitgeschichte B17: 11-22.

Tyrell, Hartmunt (1988): Institutionalisierung und Deinstitutionalisierung. In: K. Lüscher et al. (Hrsg.): Die „postmoderne" Familie. Konstanz: Universitätsverlag: 145-156.

Van de Kaa, Dirk J. (1987): Europe's Second Demographic Transition. Population Bulletin 42: $3-57$.

Vaskovics, Laszlo A. (2009): Segmentierung der Elternrolle. In G. Burkart (Hrsg.): Zukunft der Familie: Prognosen und Szenarien (Sonderheft 6 der Zeitschrift für Familienforschung). Opladen \& Farmington Hills: Barbara Budrich: 269-296.

Wagner, Michael /Franzmann, Gabriele (2000): Die Pluralisierung der Lebensformen. Zeitschrift für Bevölkerungswissenschaft 25: 151-173. 